\title{
Aspects of univalent holomorphic functions involving multiplier transformation and Ruscheweyh derivative
}

\section{Alina Alb Lupaş}

"Correspondence: dalb@uoradearo Department of Mathematics and Computer Science, University of

Oradea, str. Universitatii nr. 1,

Oradea, 410087, Romania

\begin{abstract}
Making use multiplier transformation and Ruscheweyh derivative,we introduce a new class of analytic functions $\mathcal{R} \mathcal{I}(\gamma, \lambda, l, \alpha, \beta)$ defined on the open unit disc, and investigate its various characteristics. Further we obtain distortion bounds, extreme points and radii of close-to-convexity, starlikeness and convexity and neighborhood property for functions belonging to the class $\mathcal{R} \mathcal{I}(\gamma, \lambda, l, \alpha, \beta)$.

MSC: $30 \mathrm{C} 45 ; 30 \mathrm{~A} 20 ; 34 \mathrm{~A} 40$
\end{abstract}

Keywords: analytic functions; univalent functions; radii of starlikeness and convexity; neighborhood property; Salagean operator; Ruscheweyh operator

\section{Introduction}

Let $\mathcal{A}$ denote the class of functions of the form $f(z)=z+\sum_{j=2}^{\infty} a_{j} z^{j}$, which are analytic and univalent in the open unit disc $U=\{z: z \in \mathbb{C}:|z|<1\}$. $\mathcal{T}$ is a subclass of $\mathcal{A}$ consisting of the functions of the form $f(z)=z-\sum_{j=2}^{\infty}\left|a_{j}\right| z$. For functions $f, g \in \mathcal{A}$ given by $f(z)=$ $z+\sum_{j=2}^{\infty} a_{j} z^{j}, g(z)=z+\sum_{j=2}^{\infty} b_{j} z^{j}$, we define the Hadamard product (or convolution) of $f$ and $g$ by $(f * g)(z)=z+\sum_{j=2}^{\infty} a_{j} b_{j} z^{j}, z \in U$.

Definition 1.1 (Ruscheweyh [1]) For $f \in \mathcal{A}, n \in \mathbb{N}$, the operator $R^{n}$ is defined by $R^{n}$ : $\mathcal{A} \rightarrow \mathcal{A}$

$$
\begin{aligned}
& R^{0} f(z)=f(z), \\
& R^{1} f(z)=z f^{\prime}(z), \quad \ldots, \\
& (n+1) R^{n+1} f(z)=z\left(R^{n} f(z)\right)^{\prime}+n R^{n} f(z), \quad z \in U .
\end{aligned}
$$

Remark 1.1 If $f \in \mathcal{A}, f(z)=z+\sum_{j=2}^{\infty} a_{j} z^{j}$, then $R^{n} f(z)=z+\sum_{j=2}^{\infty} \frac{(n+j-1) !}{n !(j-1) !} a_{j} z^{j}, z \in U$.

$$
\text { If } f \in \mathcal{T}, f(z)=z-\sum_{j=2}^{\infty} a_{j} z^{j} \text {, then } R^{n} f(z)=z-\sum_{j=t+1}^{\infty} \frac{(n+j-1) !}{n !(j-1) !} a_{j} z^{j}, z \in U \text {. }
$$

Definition $1.2[2,3]$ For $f \in \mathcal{A}, n \in \mathbb{N} \cup\{0\}, \lambda, l \geq 0$, the operator $I(n, \lambda, l) f(z)$ is defined by the following infinite series:

$$
I(n, \lambda, l) f(z):=z+\sum_{j=2}^{\infty}\left(\frac{\lambda(j-1)+l+1}{l+1}\right)^{n} a_{j} z^{j}
$$

(O2014 Alb Lupaş; licensee Springer. This is an Open Access article distributed under the terms of the Creative Commons Attribution License (http://creativecommons.org/licenses/by/2.0), which permits unrestricted use, distribution, and reproduction in any medium, provided the original work is properly cited. 
Remark 1.2 [4] It follows from the above definition that

$$
\begin{aligned}
& I(0, \lambda, l) f(z)=f(z) \\
& (l+1) I(n+1, \lambda, l) f(z)=(l+1-\lambda) I(n, \lambda, l) f(z)+\lambda z(I(n, \lambda, l) f(z))^{\prime}, \quad z \in U .
\end{aligned}
$$

Remark 1.3 The operator $I(n, \lambda, 0)=D_{\lambda}^{n}$ is the generalized Sălăgean operator introduced by Al-Oboudi [5], and $I(n, 1,0)=S^{n}$ is the Sălăgean differential operator [6].

Definition 1.3 $[7,8]$ Let $\gamma, \lambda, l \geq 0, n \in \mathbb{N}$. Denote by $R I_{n, \lambda, l}^{\gamma}$ the operator given by $R I_{n, \lambda, l}^{\gamma}$ : $\mathcal{A} \rightarrow \mathcal{A}$,

$$
R I_{n, \lambda, l}^{\gamma} f(z)=(1-\gamma) R^{n} f(z)+\gamma I(n, \lambda, l) f(z), \quad z \in U
$$

Remark 1.4 If $f \in \mathcal{A}, f(z)=z+\sum_{j=2}^{\infty} a_{j} z^{j}$, then

$$
\begin{aligned}
& R I_{n, \lambda, l}^{\gamma} f(z)=z+\sum_{j=2}^{\infty}\left\{\gamma\left(\frac{1+\lambda(j-1)+l}{l+1}\right)^{n}+(1-\gamma) \frac{(n+j-1) !}{n !(j-1) !}\right\} a_{j} z^{j}, \quad z \in U . \\
& \text { If } f \in \mathcal{T}, f(z)=z-\sum_{j=2}^{\infty} a_{j} z^{j} \text {, then } \\
& R I_{n, \lambda, l}^{\gamma} f(z)=z-\sum_{j=2}^{\infty}\left\{\gamma\left(\frac{1+\lambda(j-1)+l}{l+1}\right)^{n}+(1-\gamma) \frac{(n+j-1) !}{n !(j-1) !}\right\} a_{j} z^{j}, \quad z \in U .
\end{aligned}
$$

Remark 1.5 The operator $R I_{n, \lambda, 0}^{\gamma} f(z)=R D_{\lambda, \gamma}^{n} f(z)$ which was introduced in [9] and the operator $R I_{n, 1,0}^{\gamma} f(z)=L_{\gamma}^{n} f(z)$ which was introduced in [10].

Following the work of Najafzadeh and Pezeshki [11] we can define the class $\mathcal{R} \mathcal{I}(\gamma, \lambda, l$, $\alpha, \beta)$ as follows.

Definition 1.4 For $\gamma, \lambda, l \geq 0,0 \leq \alpha<1$ and $0<\beta \leq 1$, let $\mathcal{R} \mathcal{I}(\gamma, \lambda, l, \alpha, \beta)$ be the subclass of $\mathcal{T}$ consisting of functions that satisfying the inequality

$$
\left|\frac{R I_{n, \lambda, t}^{\mu, \gamma} f(z)-1}{2 v\left(R I_{n, \lambda, f}^{\mu, \gamma} f(z)-\alpha\right)-\left(R I_{n, \lambda, l}^{\mu, \gamma} f(z)-1\right)}\right|<\beta,
$$

where

$$
R I_{n, \lambda, l}^{\mu, \gamma} f(z)=(1-\mu) \frac{R I_{n, \lambda, l}^{\gamma} f(z)}{z}+\mu\left(R I_{n, \lambda, l}^{\gamma} f(z)\right)^{\prime},
$$

$0<v \leq 1$

Remark 1.6 If $f \in \mathcal{T}, f(z)=z-\sum_{j=2}^{\infty} a_{j} z^{j}$, then

$$
\begin{aligned}
R I_{n, \lambda, l}^{\mu, \gamma} f(z)=1 & -\sum_{j=t+1}^{\infty}[1+\mu(j-1)] \\
& \times\left\{\gamma\left(\frac{1+\lambda(j-1)+l}{l+1}\right)^{n}+(1-\gamma) \frac{(n+j-1) !}{n !(j-1) !}\right\} a_{j} z^{j-1}, \quad z \in U .
\end{aligned}
$$


Remark 1.7 The class $\mathcal{R} \mathcal{I}(\gamma, \lambda, 0, \alpha, \beta)=\mathcal{R} \mathcal{D}(\gamma, \lambda, \alpha, \beta)$ defined and studied in [12] and $\mathcal{R} \mathcal{I}(\gamma, 1,0, \alpha, \beta)=\mathcal{L}(\gamma, \alpha, \beta)$ defined and studied in [13].

\section{Coefficient bounds}

In this section we obtain coefficient bounds and extreme points for functions in $\mathcal{R} \mathcal{I}(\gamma, \lambda, l$, $\alpha, \beta)$.

Theorem 2.1 Let the function $f \in \mathcal{T}$. Then $f \in \mathcal{R} \mathcal{I}(\gamma, \lambda, l, \alpha, \beta)$ if and only if

$$
\begin{aligned}
& \sum_{j=t+1}^{\infty}(1+\mu(j-1))[1+\beta(2 v-1)]\left\{\gamma\left(\frac{1+\lambda(j-1)+l}{l+1}\right)^{n}+(1-\gamma) \frac{(n+j-1) !}{n !(j-1) !}\right\} a_{j} \\
& \quad<2 \beta \nu(1-\alpha) .
\end{aligned}
$$

The result is sharp for the function $F(z)$ defined by

$$
F(z)=z-\frac{2 \beta v(1-\alpha)}{(1+\mu(j-1))[1+\beta(2 v-1)]\left\{\gamma\left(\frac{1+\lambda(j-1)+l}{l+1}\right)^{n}+(1-\gamma) \frac{(n+j-1) !}{n !(j-1) !}\right\}} z^{j}, \quad j \geq t+1 .
$$

Proof Suppose $f$ satisfies (2.1). Then for $|z|<1$, we have

$$
\begin{aligned}
\left|R I_{n, \lambda}^{\mu, \gamma}, f(z)-1\right|-\beta\left|2 v\left(R I_{n, \lambda}^{\mu, \gamma} f(z)-\alpha\right)-\left(R I_{n, \lambda}^{\mu, \gamma}, f(z)-1\right)\right| & \left|-\sum_{j=t+1}^{\infty}(1+\mu(j-1))\left\{\gamma\left(\frac{1+\lambda(j-1)+l}{l+1}\right)^{n}+(1-\gamma) \frac{(n+j-1) !}{n !(j-1) !}\right\} a_{j} z^{j-1}\right| \\
& -\beta \mid 2 v(1-\alpha)-(2 v-1) \sum_{j=t+1}^{\infty}\left\{\gamma\left(\frac{1+\lambda(j-1)+l}{l+1}\right)^{n}+(1-\gamma) \frac{(n+j-1) !}{n !(j-1) !}\right\} \\
& \times[1+\mu(j-1)] a_{j} z^{j-1} \mid \\
\leq & \sum_{j=t+1}^{\infty}[1+\mu(j-1)]\left\{\gamma\left(\frac{1+\lambda(j-1)+l}{l+1}\right)^{n}+(1-\gamma) \frac{(n+j-1) !}{n !(j-1) !}\right\} a_{k}-2 \beta v(1-\alpha) \\
& +\sum_{j=t+1}^{\infty} \beta(2 v-1)(1+\mu(j-1))\left\{\gamma\left(\frac{1+\lambda(j-1)+l}{l+1}\right)^{n}+(1-\gamma) \frac{(n+j-1) !}{n !(j-1) !}\right\} a_{j} \\
= & \sum_{j=t+1}^{\infty}[1+\mu(j-1)][1+\beta(2 v-1)]\left\{\gamma\left(\frac{1+\lambda(j-1)+l}{l+1}\right)^{n}+(1-\gamma) \frac{(n+j-1) !}{n !(j-1) !}\right\} a_{j} \\
& -2 \beta v(1-\alpha)<0 .
\end{aligned}
$$

Hence, by using the maximum modulus Theorem and (1.1), $f \in \mathcal{R} \mathcal{I}(\gamma, \lambda, l, \alpha, \beta)$. Conversely, assume that

$$
\begin{aligned}
& \left|\frac{R I_{n, \lambda, t}^{\mu, \gamma} f(z)-1}{2 v\left(R I_{n, \lambda, f}^{\mu, \gamma} f(z)-\alpha\right)-\left(R I_{n, \lambda, t}^{\mu, \gamma} f(z)-1\right)}\right| \\
& \quad=\left|\frac{-\sum_{j=t+1}^{\infty}[1+\mu(j-1)]\left\{\gamma\left(\frac{1+\lambda(j-1)+l}{l+1}\right)^{n}+(1-\gamma) \frac{(n+j-1) !}{n !(j-1) !}\right\} a_{j} z^{j-1}}{2 v(1-\alpha)-\sum_{j=t+1}^{\infty}[1+\mu(j-1)](2 v-1)\left\{\gamma\left(\frac{1+\lambda(j-1)+l}{l+1}\right)^{n}+(1-\gamma) \frac{(n+j-1) !}{n !(j-1) !}\right\} a_{j} z^{j-1}}\right| \\
& \quad<\beta, \quad z \in U .
\end{aligned}
$$


Since $\operatorname{Re}(z) \leq|z|$ for all $z \in U$, we have

$$
\begin{aligned}
& \operatorname{Re}\left\{\frac{\sum_{j=t+1}^{\infty}[1+\mu(j-1)]\left\{\gamma\left(\frac{1+\lambda(j-1)+l}{l+1}\right)^{n}+(1-\gamma) \frac{(n+j-1) !}{n !(j-1) !}\right\} a_{j} z^{j-1}}{2 v(1-\alpha)-\sum_{j=t+1}^{\infty}[1+\mu(j-1)](2 v-1)\left\{\gamma\left(\frac{1+\lambda(j-1)+l}{l+1}\right)^{n}+(1-\gamma) \frac{(n+j-1) !}{n !(j-1) !}\right\} a_{j} z^{j-1}}\right\} \\
& \quad<\beta .
\end{aligned}
$$

By choosing choose values of $z$ on the real axis so that $R I_{n, \lambda, f}^{\mu, \gamma} f(z)$ is real and letting $z \rightarrow 1$ through real values, we obtain the desired inequality (2.1).

Corollary 2.2 Iff $\in \mathcal{T}$ is in $\mathcal{R} \mathcal{I}(\gamma, \lambda, l, \alpha, \beta)$, then

$$
a_{j} \leq \frac{2 \beta \nu(1-\alpha)}{[1+\mu(j-1)][1+\beta(2 \nu-1)]\left\{\gamma\left(\frac{1+\lambda(j-1)+l}{l+1}\right)^{n}+(1-\gamma) \frac{(n+j-1) !}{n !(j-1) !}\right\}}, \quad j \geq t+1,
$$

with equality only for functions of the form $F(z)$.

Theorem 2.3 Let $f_{1}(z)=z$ and

$$
\begin{aligned}
f_{j}(z) & =z-\frac{2 \beta \nu(1-\alpha)}{[1+\mu(j-1)][1+\beta(2 v-1)]\left\{\gamma\left(\frac{1+\lambda(j-1)+l}{l+1}\right)^{n}+(1-\gamma) \frac{(n+j-1) !}{n !(j-1) !}\right\}} z^{j} \\
& =t+1
\end{aligned}
$$

for $0 \leq \alpha<1,0<\beta \leq 1, \gamma, \lambda, l \geq 0$ and $0<v \leq 1$. Then $f(z)$ is in the class $\mathcal{R I}(\gamma, \lambda, l, \alpha, \beta)$ if and only if it can be expressed in the form

$$
f(z)=\sum_{j=t}^{\infty} \omega_{j} f_{j}(z)
$$

where $\omega_{j} \geq 0$ and $\sum_{j=1}^{\infty} \omega_{j}=1$.

Proof Suppose $f(z)$ can be written as in (2.5). Then

$$
f(z)=z-\sum_{j=t+1}^{\infty} \omega_{j} \frac{2 \beta \nu(1-\alpha)}{[1+\mu(j-1)][1+\beta(2 v-1)]\left\{\gamma\left(\frac{1+\lambda(j-1)+l}{l+1}\right)^{n}+(1-\gamma) \frac{(n+j-1) !}{n !(j-1) !}\right\}} z^{j} .
$$

Now,

$$
\begin{aligned}
& \sum_{j=t+1}^{\infty} \frac{[1+\mu(j-1)][1+\beta(2 v-1)]\left\{\gamma\left(\frac{1+\lambda(j-1)+l}{l+1}\right)^{n}+(1-\gamma) \frac{(n+j-1) !}{n !(j-1) !}\right\}}{2 \beta v(1-\alpha)} \omega_{j} \\
& \quad \times \frac{2 \beta v(1-\alpha)}{[1+\mu(j-1)][1+\beta(2 v-1)]\left\{\gamma\left(\frac{1+\lambda(j-1)+l}{l+1}\right)^{n}+(1-\gamma) \frac{(n+j-1) !}{n !(j-1) !}\right.}=\sum_{j=t+1}^{\infty} \omega_{j}=1-\omega_{1} \leq 1 .
\end{aligned}
$$

Thus $f \in \mathcal{R} \mathcal{I}(\gamma, \lambda, l, \alpha, \beta)$.

Conversely, let $f \in \mathcal{R} \mathcal{I}(\gamma, \lambda, l, \alpha, \beta)$. Then by using (2.3), setting

$$
\omega_{j}=\frac{[1+\mu(j-1)][1+\beta(2 v-1)]\left\{\gamma\left(\frac{1+\lambda(j-1)+l}{l+1}\right)^{n}+(1-\gamma) \frac{(n+j-1) !}{n !(j-1) !}\right\}}{2 \beta \nu(1-\alpha)} a_{j}, \quad j \geq t+1
$$


and $\omega_{1}=1-\sum_{j=2}^{\infty} \omega_{j}$, we have $f(z)=\sum_{j=t}^{\infty} \omega_{j} f_{j}(z)$. This completes the proof of Theorem 2.3 .

\section{Distortion bounds}

In this section we obtain distortion bounds for the class $\mathcal{R} \mathcal{I}(\gamma, \lambda, l, \alpha, \beta)$.

Theorem 3.1 Iff $\in \mathcal{R} \mathcal{I}(\gamma, \lambda, l, \alpha, \beta)$, then

$$
\begin{aligned}
& r-\frac{2 \beta \nu(1-\alpha)}{(1+\mu t)[1+\beta(2 v-1)]\left\{\gamma\left(\frac{1+\lambda t+l}{l+1}\right)^{n}+(1-\gamma) \frac{(n+t) !}{n ! t !}\right\}} r^{t+1} \\
& \quad \leq|f(z)| \leq r+\frac{2 \beta \nu(1-\alpha)}{(1+\mu t)[1+\beta(2 v-1)]\left\{\gamma\left(\frac{1+\lambda t+l}{l+1}\right)^{n}+(1-\gamma) \frac{(n+t) !}{n ! t !}\right\}} r^{t+1}
\end{aligned}
$$

holds if the sequence $\left\{\sigma_{j}(\gamma, \lambda, l, \beta, \nu)\right\}_{j=t+1}^{\infty}$ is non-decreasing, and

$$
\begin{aligned}
& 1-\frac{2 \beta v(1-\alpha)(t+1)}{(1+\mu t)[1+\beta(2 v-1)]\left\{\gamma\left(\frac{1+\lambda t+l}{l+1}\right)^{n}+(1-\gamma) \frac{(n+t) !}{n ! t !}\right\}} r^{t} \\
& \leq\left|f^{\prime}(z)\right| \leq 1+\frac{2 \beta \nu(1-\alpha)(t+1)}{(1+\mu t)[1+\beta(2 v-1)]\left\{\gamma\left(\frac{1+\lambda t+l}{l+1}\right)^{n}+(1-\gamma) \frac{(n+t) !}{n ! t !}\right\}} r^{t}
\end{aligned}
$$

holds if the sequence $\left\{\frac{\sigma_{j}(\gamma, \lambda, l, \beta, \nu)}{j}\right\}_{j=t+1}^{\infty}$ is non-decreasing, where

$$
\sigma_{j}(\gamma, \beta, \nu)=[1+\mu(j-1)][1+\beta(2 v-1)]\left\{\gamma\left(\frac{1+\lambda(j-1)+l}{l+1}\right)^{n}+(1-\gamma) \frac{(n+j-1) !}{n !(j-1) !}\right\}
$$

The bounds in (3.1) and (3.2) are sharp, for $f(z)$ given by

$$
f(z)=z-\frac{2 \beta v(1-\alpha)}{(1+\mu t)[1+\beta(2 v-1)]\left\{\gamma\left(\frac{1+\lambda t+l}{l+1}\right)^{n}+(1-\gamma) \frac{(n+t) !}{n ! t !}\right\}} z^{t+1}, \quad z= \pm r
$$

Proof In view of Theorem 2.1, we have

$$
\sum_{j=t+1}^{\infty} a_{j} \leq \frac{2 \beta v(1-\alpha)}{(1+\mu t)[1+\beta(2 v-1)]\left\{\gamma\left(\frac{1+\lambda t+l}{l+1}\right)^{n}+(1-\gamma) \frac{(n+t) !}{n ! t !}\right\}}
$$

We obtain

$$
|z|-|z|^{t+1} \sum_{j=t+1}^{\infty} a_{j} \leq|f(z)| \leq|z|+|z|^{t+1} \sum_{j=t+1}^{\infty} a_{j}
$$

Thus

$$
\begin{aligned}
& r-\frac{2 \beta v(1-\alpha)}{(1+\mu t)[1+\beta(2 v-1)]\left\{\gamma\left(\frac{1+\lambda t+l}{l+1}\right)^{n}+(1-\gamma) \frac{(n+t) !}{n ! t !}\right\}} r^{t+1} \\
& \quad \leq|f(z)| \leq r+\frac{2 \beta \nu(1-\alpha)}{(1+\mu t)[1+\beta(2 v-1)]\left\{\gamma\left(\frac{1+\lambda t+l}{l+1}\right)^{n}+(1-\gamma) \frac{(n+t) !}{n ! t !}\right\}} r^{t+1} .
\end{aligned}
$$


Hence (3.1) follows from (3.5). Further,

$$
\sum_{j=t+1}^{\infty} j a_{j} \leq \frac{2 \beta \nu(1-\alpha)}{(1+\mu t)[1+\beta(2 v-1)]\left\{\gamma\left(\frac{1+\lambda t+l}{l+1}\right)^{n}+(1-\gamma) \frac{(n+t) !}{n ! t !}\right\}}
$$

Hence (3.2) follows from

$$
1-r^{t} \sum_{j=t+1}^{\infty} j a_{j} \leq\left|f^{\prime}(z)\right| \leq 1+r^{t} \sum_{j=t+1}^{\infty} j a_{j}
$$

\section{Radius of starlikeness and convexity}

The radii of close-to-convexity, starlikeness, and convexity for the class $\mathcal{R} \mathcal{I}(\gamma, \lambda, l, \alpha, \beta)$ are given in this section.

Theorem 4.1 Let the function $f \in \mathcal{T}$ belong to the class $\mathcal{R} \mathcal{I}(\gamma, \lambda, l, \alpha, \beta)$, Then $f(z)$ is closeto-convex of order $\delta, 0 \leq \delta<1$, in the disc $|z|<r$, where

$$
r:=\inf _{j \geq t+1}\left[\frac{(1-\delta)[1+\mu(j-1)][1+\beta(2 v-1)]\left\{\gamma\left(\frac{1+\lambda(j-1)+l}{l+1}\right)^{n}+(1-\gamma) \frac{(n+j-1) !}{n !(j-1) !}\right\}}{2 \beta v j(1-\alpha)}\right]^{\frac{1}{t}} .
$$

The result is sharp, with extremal function $f(z)$ given by (2.3).

Proof For given $f \in \mathcal{T}$ we must show that

$$
\left|f^{\prime}(z)-1\right|<1-\delta
$$

By a simple calculation we have

$$
\left|f^{\prime}(z)-1\right| \leq \sum_{j=t+1}^{\infty} j a_{j}|z|^{t}
$$

The last expression is less than $1-\delta$ if

$$
\sum_{j=t+1}^{\infty} \frac{j}{1-\delta} a_{j}|z|^{t}<1
$$

We use the fact that $f \in \mathcal{R} \mathcal{I}(\gamma, \lambda, l, \alpha, \beta)$ if and only if

$$
\sum_{j=t+1}^{\infty} \frac{[1+\mu(j-1)][1+\beta(2 v-1)]\left\{\gamma\left(\frac{1+\lambda(j-1)+l}{l+1}\right)^{n}+(1-\gamma) \frac{(n+j-1) !}{n !(j-1) !}\right\}}{2 \beta v(1-\alpha)} a_{j} \leq 1
$$

Equation (4.2) holds true if

$$
\frac{j}{1-\delta}|z|^{t} \leq \sum_{j=t+1}^{\infty} \frac{[1+\mu(j-1)][1+\beta(2 v-1)]\left\{\gamma\left(\frac{1+\lambda(j-1)+l}{l+1}\right)^{n}+(1-\gamma) \frac{(n+j-1) !}{n !(j-1) !}\right\}}{2 \beta \nu(1-\alpha)}
$$


Or, equivalently,

$$
|z|^{t} \leq \sum_{j=t+1}^{\infty} \frac{(1-\delta)[1+\mu(j-1)][1+\beta(2 v-1)]\left\{\gamma\left(\frac{1+\lambda(j-1)+l}{l+1}\right)^{n}+(1-\gamma) \frac{(n+j-1) !}{n !(j-1) !}\right\}}{2 \beta v j(1-\alpha)},
$$

which completes the proof.

Theorem 4.2 Let $f \in \mathcal{R} \mathcal{I}(\gamma, \lambda, l, \alpha, \beta)$. Then

1. $f$ is starlike of order $\delta, 0 \leq \delta<1$, in the disc $|z|<r_{1}$, where

$$
r_{1}=\inf _{j \geq t+1}\left\{\frac{(1-\delta)[1+\mu(j-1)][1+\beta(2 v-1)]\left\{\gamma\left(\frac{1+\lambda(j-1)+l}{l+1}\right)^{n}+(1-\gamma) \frac{(n+j-1) !}{n !(j-1) !}\right\}}{2 \beta v(1-\alpha)(j-\delta)}\right\}^{\frac{1}{t}} .
$$

2. $f$ is convex of order $\delta, 0 \leq \delta<1$, in the disc $|z|<r_{2}$ where,

$$
r_{2}=\inf _{j \geq t+1}\left\{\frac{(1-\delta)[1+\mu(j-1)][1+\beta(2 v-1)]\left\{\gamma\left(\frac{1+\lambda(j-1)+l}{l+1}\right)^{n}+(1-\gamma) \frac{(n+j-1) !}{n !(j-1) !}\right\}}{2 \beta v j(j-1)(1-\alpha)}\right\}^{\frac{1}{t}} .
$$

Each of these results is sharp for the extremal function $f(z)$ given by (2.5).

Proof 1 . For $0 \leq \delta<1$ we need to show that

$$
\left|\frac{z f^{\prime}(z)}{f(z)}-1\right|<1-\delta \text {. }
$$

We have

$$
\left|\frac{z f^{\prime}(z)}{f(z)}-1\right| \leq\left|\frac{\sum_{j=t+1}^{\infty}(j-1) a_{j}|z|^{t}}{1-\sum_{j=t+1}^{\infty} a_{j}|z|^{t}}\right| .
$$

The last expression is less than $1-\delta$ if

$$
\sum_{j=t+1}^{\infty} \frac{(j-\delta)}{1-\delta} a_{j}|z|^{t}<1
$$

We use the fact that $f \in \mathcal{R} \mathcal{I}(\gamma, \lambda, l, \alpha, \beta)$ if and only if

$$
\sum_{j=t+1}^{\infty} \frac{[1+\mu(j-1)][1+\beta(2 v-1)]\left\{\gamma\left(\frac{1+\lambda(j-1)+l}{l+1}\right)^{n}+(1-\gamma) \frac{(n+j-1) !}{n !(j-1) !}\right\}}{2 \beta v(1-\alpha)} a_{j}<1 .
$$

Equation (4.3) holds true if

$$
\frac{j-\delta}{1-\delta}|z|^{t}<\frac{[1+\mu(j-1)][1+\beta(2 v-1)]\left\{\gamma\left(\frac{1+\lambda(j-1)+l}{l+1}\right)^{n}+(1-\gamma) \frac{(n+j-1) !}{n !(j-1) !}\right\}}{2 \beta v(1-\alpha)} .
$$

Or, equivalently,

$$
|z|^{t}<\frac{(1-\delta)[1+\mu(j-1)][1+\beta(2 v-1)]\left\{\gamma\left(\frac{1+\lambda(j-1)+l}{l+1}\right)^{n}+(1-\gamma) \frac{(n+j-1) !}{n !(j-1) !}\right\}}{2 \beta v(1-\alpha)(j-\delta)},
$$

which yields the starlikeness of the family. 
2. Using the fact that $f$ is convex if and only $z f^{\prime}$ is starlike, we can prove (2) with a similar way of the proof of (1). The function $f$ is convex if and only if

$$
\left|z f^{\prime \prime}(z)\right|<1-\delta
$$

We have

$$
\begin{aligned}
& \left|z f^{\prime \prime}(z)\right| \leq\left.\left|\sum_{j=t+1}^{\infty} j(j-1) a_{j}\right| z\right|^{t-1} \mid<1-\delta, \\
& \sum_{j=t+1}^{\infty} \frac{j(j-1)}{1-\delta} a_{j}|z|^{t-1}<1 .
\end{aligned}
$$

We use the fact that $f \in \mathcal{R} \mathcal{I}(\gamma, \lambda, l, \alpha, \beta)$ if and only if

$$
\sum_{j=t+1}^{\infty} \frac{[1+\mu(j-1)][1+\beta(2 \nu-1)]\left\{\gamma\left(\frac{1+\lambda(j-1)+l}{l+1}\right)^{n}+(1-\gamma) \frac{(n+j-1) !}{n !(j-1) !}\right\}}{2 \beta \nu(1-\alpha)} a_{j}<1 .
$$

Equation (4.4) holds true if

$$
\frac{j(j-1)}{1-\delta}|z|^{t-1}<\frac{[1+\mu(j-1)][1+\beta(2 \nu-1)]\left\{\gamma\left(\frac{1+\lambda(j-1)+l}{l+1}\right)^{n}+(1-\gamma) \frac{(n+j-1) !}{n !(j-1) !}\right\}}{2 \beta \nu(1-\alpha)},
$$

or, equivalently,

$$
|z|^{t-1}<\frac{(1-\delta)[1+\mu(j-1)][1+\beta(2 v-1)]\left\{\gamma\left(\frac{1+\lambda(j-1)+l}{l+1}\right)^{n}+(1-\gamma) \frac{(n+j-1) !}{n !(j-1) !}\right\}}{2 \beta \nu j(j-1)(1-\alpha)},
$$

which yields the convexity of the family.

\section{Neighborhood property}

In this section we study neighborhood property for functions in the class $\mathcal{R} \mathcal{I}(\gamma, \lambda, l, \alpha, \beta)$.

Definition 5.1 For functions $f$ belong to $\mathcal{A}$ of the form and $\varepsilon \geq 0$, we define $(\eta-\varepsilon)$ neighborhood of $f$ by

$$
N_{\varepsilon}^{\eta}(f)=\left\{g(z) \in \mathcal{A}: g(z)=z+\sum_{j=2}^{\infty} b_{j} z^{j}, \sum_{j=2}^{\infty} j^{\eta+1}\left|a_{j}-b_{j}\right| \leq \varepsilon\right\},
$$

where $\eta$ is a fixed positive integer.

By using the following lemmas we will investigate the $(\eta-\varepsilon)$-neighborhood of function in $\mathcal{R} \mathcal{I}(\gamma, \lambda, l, \alpha, \beta)$.

Lemma 5.1 Let $-1 \leq \beta<1$, if $g(z)=z+\sum_{j=2}^{\infty} b_{j} z^{j}$ satisfies

$$
\sum_{j=2}^{\infty} j^{\rho+1}\left|b_{j}\right| \leq \frac{2 \beta \nu(1-\alpha)}{1+\beta(2 v-1)}
$$

then $g(z) \in \mathcal{R} \mathcal{I}(\gamma, \lambda, l, \alpha, \beta)$. 
Proof By using of Theorem 2.1, it is sufficient to show that

$$
\frac{[1+\mu(j-1)][1+\beta(2 v-1)]\left\{\gamma\left(\frac{1+\lambda(j-1)+l}{l+1}\right)^{\rho}+(1-\gamma) \frac{(\rho+j-1) !}{\rho !(j-1) !}\right\}}{2 \beta v(1-\alpha)}=\frac{j^{\rho+1}}{2 \beta \nu(1-\alpha)}[1+\beta(2 v-1)] .
$$

But

$$
\frac{[1+\beta(2 v-1)]\left\{\gamma\left(\frac{1+\lambda(j-1)+l}{l+1}\right)^{\rho}+(1-\gamma) \frac{(\rho+j-1) !}{\rho !(j-1) !}\right\}}{2 \beta \nu(1-\alpha)} \leq \frac{j^{\rho+1}}{2 \beta \nu(1-\alpha)}[1+\beta(2 v-1)]
$$

Therefore it is enough to prove that

$$
Q(j, \rho)=\frac{\gamma\left(\frac{1+\lambda(j-1)+l}{l+1}\right)^{\rho}+(1-\gamma) \frac{(\rho+j-1) !}{\rho !(j-1) !}}{j^{\rho+1}} \leq 1,
$$

the result follows because the last inequality holds for all $j \geq t+1$.

Lemma 5.2 Let $f(z)=z-\sum_{k=2}^{\infty} a_{k} z^{k} \in \mathcal{T}, \gamma, \lambda, l \geq 0,0 \leq \alpha<1,0<\beta \leq 1$ and $\varepsilon \geq 0$. If $\frac{f(z)+\epsilon z}{1+\epsilon} \in \mathcal{R} \mathcal{I}(\gamma, \lambda, l, \alpha, \beta)$, then

$$
\sum_{j=t+1}^{\infty} j^{\rho+1} a_{j} \leq \frac{2 \beta \nu(1-\alpha)(1+\epsilon)(t+1)^{\rho+1}}{[1+\mu(j-1)][1+\beta(2 v-1)]\left\{\gamma\left(\frac{1+\lambda t+l}{l+1}\right)^{n}+(1-\gamma) \frac{(n+t) !}{n ! t !}\right\}}
$$

where either $\rho=0$ or $\rho=1$. The result is sharp with the extremal function

$$
f(z)=z-\frac{2 \beta \nu(1-\alpha)(1+\epsilon)}{[1+\mu(j-1)][1+\beta(2 v-1)]\left\{\gamma\left(\frac{1+\lambda t+l}{l+1}\right)^{n}+(1-\gamma) \frac{(n+t) !}{n ! t !}\right\}} z^{t+1}, \quad z \in U
$$

Proof Letting $g(z)=\frac{f(z)+\epsilon z}{1+\epsilon}$ we have

$$
g(z)=z-\sum_{j=t+1}^{\infty} \frac{a_{j}}{1+\epsilon} z^{j}, \quad z \in U
$$

In view of Theorem $2.3, g(z)=\sum_{j=1}^{\infty} \eta_{j} g_{j}(z)$ where $\eta_{j} \geq 0, \sum_{j=1}^{\infty} \eta_{j}=1$,

$$
g_{1}(z)=z
$$

and

$$
g_{j}(z)=z-\frac{2 \beta \nu(1-\alpha)(1+\epsilon)}{[1+\mu(j-1)][1+\beta(2 v-1)]\left\{\gamma\left(\frac{1+\lambda(j-1)+l}{l+1}\right)^{n}+(1-\gamma) \frac{(n+j-1) !}{n !(j-1) !}\right\}} z^{j}, \quad j \geq t+1 .
$$

So we obtain

$$
\begin{aligned}
g(z) & =\eta_{1} z+\sum_{j=t+1}^{\infty} \eta_{j}\left[z-\frac{2 \beta v(1-\alpha)(1+\epsilon)}{[1+\mu(j-1)][1+\beta(2 v-1)]\left\{\gamma\left(\frac{1+\lambda(j-1)+l}{l+1}\right)^{n}+(1-\gamma) \frac{(n+j-1) !}{n !(j-1) !}\right\}} z^{j}\right] \\
& =z-\sum_{j=t+1}^{\infty} \eta_{k} \frac{2 \beta \nu(1-\alpha)(1+\epsilon)}{[1+\mu(j-1)][1+\beta(2 v-1)]\left\{\gamma\left(\frac{1+\lambda(j-1)+l}{l+1}\right)^{n}+(1-\gamma) \frac{(n+j-1) !}{n !(j-1) !}\right\}} z^{j} .
\end{aligned}
$$


Since $\eta_{j} \geq 0$ and $\sum_{j=2}^{\infty} \eta_{j} \leq 1$, it follows that

$$
\sum_{j=t+1}^{\infty} a_{k} \leq \sup _{j \geq t+1} j^{\rho+1} \frac{2 \beta \nu(1-\alpha)(1+\epsilon)}{[1+\mu(j-1)][1+\beta(2 v-1)]\left\{\gamma\left(\frac{1+\lambda(j-1)+l}{l+1}\right)^{n}+(1-\gamma) \frac{(n+j-1) !}{n !(j-1) !}\right\}} .
$$

Since whenever $\rho=0$ or $\rho=1$ we conclude that

$$
W(j, \rho, \gamma, \alpha, \beta, \epsilon)=j^{\rho+1} \frac{2 \beta \nu(1-\alpha)(1+\epsilon)}{[1+\mu(j-1)][1+\beta(2 \nu-1)]\left\{\gamma\left(\frac{1+\lambda(j-1)+l}{l+1}\right)^{n}+(1-\gamma) \frac{(n+j-1) !}{n !(j-1) !}\right\}}
$$

is a decreasing function of $j$, the result will follow. The proof is complete.

Theorem 5.1 Let $\rho=0$ or $\rho=1$ and suppose $0 \leq \beta<1$ and

$$
\begin{aligned}
-1 & \leq \theta \\
& <\frac{[1+\mu(t-1)][1+\beta(2 v-1)]\left\{\gamma\left(\frac{1+\lambda t+l}{l+1}\right)^{n}+(1-\gamma) \frac{(n+t) !}{n ! t !}\right\}-2 \beta v(1-\alpha)(1+\epsilon)(t+1)^{\eta+1}}{[1+\mu(t-1)][1+\beta(2 v-1)]\left\{\gamma\left(\frac{1+\lambda t+l}{l+1}\right)^{n}+(1-\gamma) \frac{(n+t) !}{n ! t !}\right\}},
\end{aligned}
$$

$f(z) \in \mathcal{T}$ and $\frac{f(z)+\epsilon z}{1+\epsilon} \in \mathcal{R I}(\gamma, \lambda, l, \alpha, \beta)$, then the $(\eta-\varepsilon)$-neighborhood of $f$ is the subset of $\mathcal{R} \mathcal{I}(\lambda, \lambda, l, \alpha, \beta)$, where

$$
\begin{aligned}
\varepsilon \leq & 2(1-\alpha)\left\{\theta \gamma[1+\mu(t-1)][1+\beta(2 v-1)]\left\{\gamma\left(\frac{1+\lambda t+l}{l+1}\right)^{n}+(1-\gamma) \frac{(n+t) !}{n ! t !}\right\}\right. \\
& \left.-\beta \gamma[1+\theta(2 v-1)](1+\epsilon)(t+1)^{\eta+1}\right\} /([1+\theta(2 v-1)][1+\mu(t-1)] \\
& \left.\times[1+\beta(2 v-1)]\left\{\gamma\left(\frac{1+\lambda t+l}{l+1}\right)^{n}+(1-\gamma) \frac{(n+t) !}{n ! t !}\right\}\right) .
\end{aligned}
$$

The result is sharp.

Proof For $f(z)=z-\sum_{j=2}^{\infty}\left|a_{j}\right| z^{j}$, let $g(z)=z+\sum_{j=2}^{\infty} b_{j} z^{j}$ be in $N_{\varepsilon}^{\eta}(f)$. So by Lemma 5.2 , we have

$$
\begin{aligned}
\sum_{j=2}^{\infty} j^{\eta+1}\left|b_{j}\right| & =\sum_{j=2}^{\infty} j^{\eta+1}\left|a_{j}-b_{j}-a_{j}\right| \\
& \leq \varepsilon+\frac{2 \beta \nu(1-\alpha)(1+\epsilon)(t+1)^{\eta+1}}{[1+\mu(t-1)][1+\beta(2 v-1)]\left\{\gamma\left(\frac{1+\lambda t+l}{l+1}\right)^{n}+(1-\gamma) \frac{(n+t) !}{n ! t !}\right\}}
\end{aligned}
$$

By using Lemma $5.1, g(z) \in \mathcal{L}(\gamma, \alpha, \beta)$ if

$$
\varepsilon+\frac{2 \beta \nu(1-\alpha)(1+\epsilon)(t+1)^{\eta+1}}{[1+\mu(t-1)][1+\beta(2 v-1)]\left\{\gamma\left(\frac{1+\lambda t+l}{l+1}\right)^{n}+(1-\gamma) \frac{(n+t) !}{n ! t !}\right\}} \leq \frac{2 \theta v(1-\alpha)}{1+\theta(2 v-1)},
$$

that is, $\varepsilon \leq \frac{2(1-\alpha)\left\{\theta \gamma[1+\mu(t-1)][1+\beta(2 v-1)]\left\{\gamma\left(\frac{1+\lambda t+l}{l+1}\right)^{n}+(1-\gamma) \frac{(n+t) !}{n ! t}\right\}-\beta \gamma[1+\theta(2 v-1)](1+\epsilon)(t+1)^{\eta+1}\right\}}{[1+\theta(2 v-1)][1+\mu(t-1)][1+\beta(2 v-1)]\left\{\gamma\left(\frac{1+\lambda t+l}{l+1}\right)^{n}+(1-\gamma) \frac{(n+t) !}{n ! t !}\right\}}$, and the proof is complete. 


\section{Competing interests}

The author declares that she has no competing interests.

\section{Author's contributions}

The author drafted the manuscript, read and approved the final manuscript.

Received: 1 March 2014 Accepted: 9 April 2014 Published: 06 May 2014

\section{References}

1. Ruscheweyh, S: New criteria for univalent functions. Proc. Am. Math. Soc. 49, 109-115 (1975)

2. Alb Lupaş, A: A special comprehensive class of analytic functions defined by multiplier transformation. J. Comput. Anal. Appl. 12(2), 387-395 (2010)

3. Alb Lupaş, A: A new comprehensive class of analytic functions defined by multiplier transformation. Math. Comput. Model. 54, 2355-2362 (2011). doi:10.1016/j.mcm.2011.05.044

4. Alb Lupaş, A: On special differential superordinations using multiplier transformation. J. Comput. Anal. Appl. 13(1), 121-126 (2011)

5. Al-Oboudi, FM: On univalent functions defined by a generalized Sălăgean operator. Int. J. Math. Math. Sci. 27, 1429-1436 (2004)

6. Sălăgean, GS: Subclasses of univalent functions. In: Lecture Notes in Math., vol. 1013, pp. 362-372. Springer, Berlin (1983)

7. Alb Lupaş, A: On special differential subordinations using multiplier transformation and Ruscheweyh derivative. Romai J. 6(2), 1-14 (2010)

8. Alb Lupaş, A: Certain special differential superordinations using multiplier transformation and Ruscheweyh derivative. J. Comput. Anal. Appl. 13(1), 108-115 (2011)

9. Alb Lupaş, A: On special differential subordinations using a generalized Sălăgean operator and Ruscheweyh derivative. J. Comput. Anal. Appl. 13(1), 98-107 (2011)

10. Alb Lupaş, A: On special differential subordinations using Sălăgean and Ruscheweyh operators. Math. Inequal. Appl. 12(4), 781-790 (2009)

11. Najafzadeh, S, Pezeshki, E: Some aspects of univalent holomorphic functions involving Ruscheweyh and Salagean operator. An. Univ. Oradea, Fasc. Mat. XX(1), 61-70 (2013)

12. Alb Lupas, A, Andrei, L: Aspects of univalent holomorphic functions involving Ruscheweyh derivative and generalized Sălăgean operator. J. Comput. Anal. Appl. (to appear)

13. Alb Lupas, A: Aspects of univalent holomorphic functions involving Sălăgean operator and Ruscheweyh derivative. J. Concr. Appl. Math. (to appear)

10.1186/1687-1847-2014-117

Cite this article as: Alb Lupaş: Aspects of univalent holomorphic functions involving multiplier transformation and Ruscheweyh derivative. Advances in Difference Equations 2014, 2014:117

\section{Submit your manuscript to a SpringerOpen ${ }^{\ominus}$ journal and benefit from:}

- Convenient online submission

- Rigorous peer review

- Immediate publication on acceptance

- Open access: articles freely available online

- High visibility within the field

- Retaining the copyright to your article 